\title{
A Novel, Fast and Efficient One-Pot Four-Component Procedure for Preparation of Some Spiro[indeno [1,2-b]quinoxaline-11,3'-pyrrolizidine]
}

\author{
Mohammad R. Mohammadizadeh' and Neda Fiıozi \\ Department of Chemistrv, Facultw of Sciences, Persian Gulf Cniversitv. Bushehr 75169, Iran \\ ${ }^{\star}$ E-mail: mrmohamadizadehapgu.acir \\ Received April 27, 2009. Accepted June 23, 2009
}

Key Worls: Multicomponent reaction. Ninhỵ'drin. Phenylenediamines. Proline. Spiro[indeno[1.2-b]quinoxaline-11,3'-pyrrolizidine]

Pyrrolizine and indolizine skeletons, partially or totally saturated, are present in a large array of alkaloids and related unnatural compounds. Such compounds exhibit potent biological activities. particularly as glycosidase inhibitors. ${ }^{3}$ The cycloaddition of azomethine ylides to alkenes is an important way to synthesize heterocycles containing py'rrolidine substrnictures with high stereoselectivity. The stereoselectivity of these cycloaddition reactions is greatly enhanced if the azomethine ylide functionality is part of an N-heterocycle. thus providing a rather rigid ring template those results in a better diastereofacial approach between dipolar and dipolarophile. Cyclic azomethine ylides, in which the central nitrogen atom is part of a pyrrolidine ring. are of particular importance. since they can be directly transformed into pyrolizidine rings through a cycloaddition reaction with alkenes in a highly stereoselective way. ${ }^{3}$

In recent years multicomponent reactions (MCRs) have been extensively studied due to their efficiency. atom economy and convenience in construction of multiple new bonds in one pot processes. which played as powerful roles in approach to complex structures and promoted the "green chemistry". In continuing our interest in synthesis of nitrogen containing heterocycles" and as a part of our ongoing programs on ninhydrin-based multi-component reactions, ${ }^{6}$ and due to the resultant pharmacological interest in py rrolizidine alkaloids. here we report a novel one-pot reaction which affords alkyl spiro[indeno[1.2-b]quinoxaline-11.3'-py rrolizidine] 5 via the four-component condensation of ninhydrin 1, phenylenediamine 2. proline 3. and chalcone 4 in ethanol in very good yields (Scheme 1)

The one-pot four-component reaction proceeds fast and very cleanly at reflux temperature and no undesirable side reactions were observed. The results are shown in Table 1.
The isolated spiro[indeno[1.2-b]quinoxaline-11.3'-pyrrolizine]$2^{\prime}$-carboxylates 5 were characterized on the basis of IR, ${ }^{1} \mathrm{H}$ and ${ }^{13} \mathrm{C}$ NMR spectroscopy and elemental analysis. The IR spectrum of 5a shows absorption at $1695 \mathrm{~cm}^{-1}$ indicating the presence of carbonyl functionality. The ${ }^{1} \mathrm{H}$ NMR spectnum of 5 a exhibited characteristic multiplets of py rrolizidine ring at $\delta$ 1.92 - 2.72. Additionally. ${ }^{\mathrm{l}} \mathrm{H}$ NMR spectra of 5a shown characteristic triplet at $\hat{\delta} 4.24$. doublet of triplet at $\hat{\delta} 4.51$ and doublets at $\delta 5.47$ for protons $\mathrm{H}^{\mathrm{j}^{\prime}}$. $\mathrm{H}^{\mathrm{F}^{\prime \prime}}$ and $\mathrm{H}^{2}$ respectively. It is noticible. if the signal at 4.24 was irradiated. peaks of $\mathrm{H}^{* \prime a}$ and $\mathrm{H}^{2 \prime}$ change to triplet and singlet, respectively. In the ${ }^{15} \mathrm{C}$ NMR spectrum of 5a. the spiro carbon and ester's carbonyl are resonated at $\bar{\delta} 75.8$ and 198.2 respectively and the signals for all other 27 carbons are located at appropriate chemical shifts in agreement with the proposed structure.

The formation of the spiro[indeno[1,2-b]quinoxaline- 11 . 3'-pyrrolizine] scaffold probably involves a complex multistep sequence of events. On the basis of the well known chemistry of ninhydrin and indenoquinoxaline, mechanistically. it is

Table 1. One-pot synthesis of spiro[indeno[1,2-b]quinosaline-11,3'pyrrolizidine 5a-f

\begin{tabular}{clllc}
\hline $\mathbf{5}^{a b}$ & $\mathrm{R}^{\mathrm{l}}$ & $\mathrm{R}^{2}$ & $\mathrm{R}^{3}$ & Yield $^{c}$ \\
\hline $\mathbf{a}$ & $\mathrm{H}$ & $\mathrm{H}$ & $\mathrm{H}$ & 75 \\
$\mathbf{b}$ & $\mathrm{Me}$ & $\mathrm{H}$ & $\mathrm{H}$ & 80 \\
$\mathbf{c}$ & $\mathrm{Me}$ & $\mathrm{Me}$ & $\mathrm{H}$ & 77 \\
$\mathbf{d}$ & $\mathrm{H}$ & $\mathrm{F}$ & $\mathrm{H}$ & 75 \\
$\mathbf{e}$ & $\mathrm{Me}$ & $\mathrm{H}$ & $\mathrm{F}$ & 80 \\
$\mathbf{f}$ & $\mathrm{Me}$ & $\mathrm{F}$ & $\mathrm{F}$ & 82
\end{tabular}

Ninhydrin:diamine:proline:chalcone ( $1 \mathrm{mmol}: 1 \mathrm{mmol}: 1 \mathrm{mmol}: 1 \mathrm{mmol}$ ): "The structure of products 5 a-f were fully characterized by nmin and mass spectroscopv: 'All referred to isolated pure products.

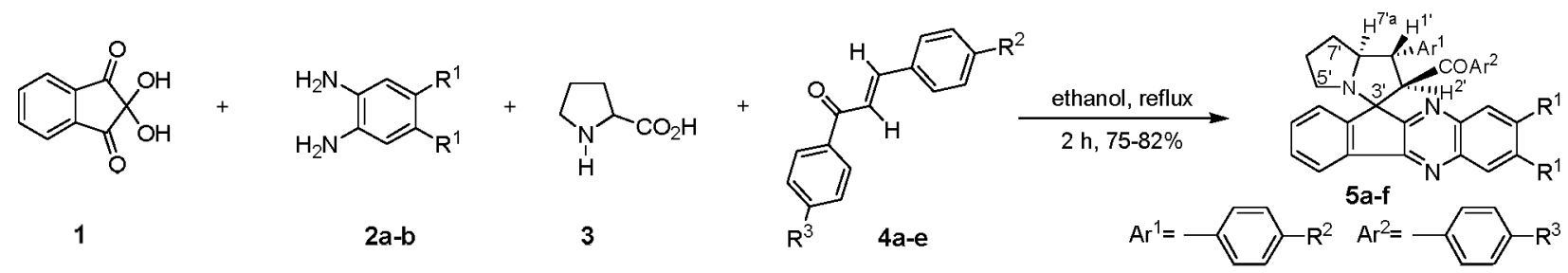

Scheme 1. One-pot four-component stereoslective synthesis of spiro[indeno[1,2-b]quinoxaline-11,3'-pyrrolizidine] 

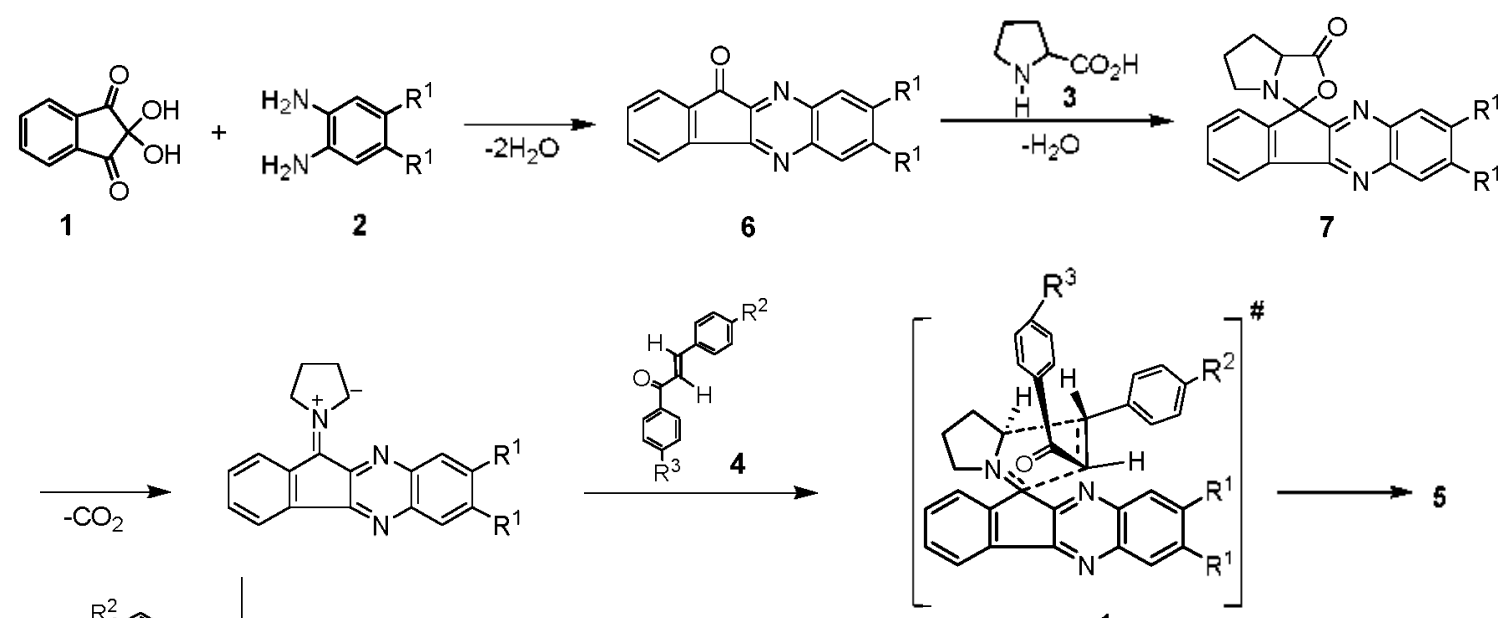

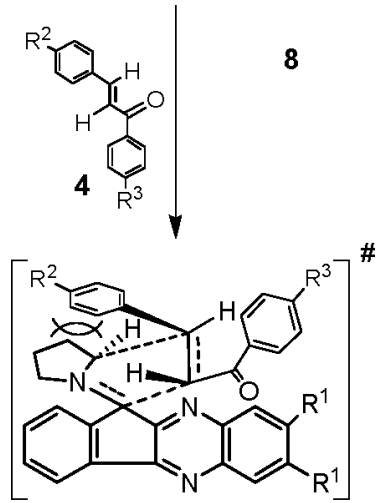

$\mathrm{TS}^{2}$

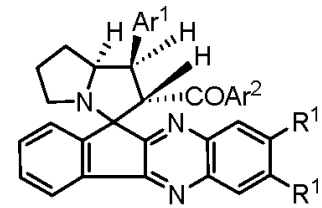

5'

Scheme 2. The proposed mechanism for the one-pot four-component stereoslective preparation of spiro[indeno[1,2-b]quinoxaline-11,3'pynolizidine]

reasonable to assume that initial condensation of the phenylenediamine 2 and the ninlyydrin 1 gives indenoquinosline-1 1one intermediate 6 . which condensed with proline 3 to produce 1.3-dipolar azomethine ylides $8^{8}$ The formed 1.3-dipolar species $\mathbf{8}$ subsequently undergoes cycloaddition reaction with chalcone 4 , to produce stereoselectively the new adduct 5 (Scheme 2).

According to the presence of carbonyl group as a spacer. $\mathrm{R}^{3}$ substituted phenyl ring can get sufficiently away from the indenoquinoxaline structure, therefore $\mathrm{TS}^{\mathrm{j}^{3}}$ has lower strike hindrance compared to $\mathrm{TS}^{2}$, in which the $\mathrm{R}^{2}$ substituted phenyl ring is substantially close to proline moiety. Consequently. the reaction proceeds via $\mathrm{TS}^{1}$. In practice, almost stereoisomer 5 was obtained. without obtaining any stereoisomer 5'.

High diastereomeric excess of reaction was deduced on the basis of ${ }^{1} \mathrm{H}$ NMR spectra through which no other diastereomers could be detected. It is noteworthy that adducts 5 have three or four (concerning nitrogen) chiral centers. but their synthesis affords only one diastereomer, due to the fixed configuration of dipole $\mathbf{8}$ and the stnicture of transition state 1 $\left(\mathrm{TS}^{\mathrm{j}}\right.$ ), that has been mentioned by Grigg and his co-workers later, in their extensive studies. ${ }^{9}$ Stereochemical assignments of the cycloadducts 5 a are made on the basis of N.O.E. difference spectroscopy and comparison with previously reported relative systems. The possibility of the formation of other isomer wia $\mathrm{TS}^{2}$. was ruled out by ${ }^{3} \mathrm{H}$ NOESY studies. For example irradia- tion of $\mathrm{H}^{\mathrm{l}^{\prime}}$ at $\delta=4.24$ caused no considerable enthancement of $\mathrm{H}^{2 \prime}$ and $\mathrm{H}^{\prime \prime \prime}$ at $\delta=4.5 \mathrm{l}$ and 5.47 , respectively, supporting the nutual trans arrangenent of $\mathrm{H}^{-i^{\prime} \mathrm{a}}$ and $\mathrm{H}^{\mathrm{l}^{\prime}}$.

However. the products can be achieved by a two-step procedure: first. the indenoquinoxaline 6 was syinthesized and purified from the reaction of ninhydrin 1 and aromatic diamine 2. and then the final product 5 was prepared via a three-component reaction between indenoquinoxaline 6 . proline 3 and chalcone 4. Using this two step procedure the overall yield for preparation of $2^{\prime}$-benzoyl-1'.2'.5',6'.7',7'a-hexalyydro-1'-phenylspiro[indeno[1,2-b]quinoxaline-11,3'-py rrolizine] 5a is about $67 \%$. which much less than the one-pot four-component procedure. Moreover. many times and solvents were saved using the one-pot four-component procedure

In sunumary. our study introduced a new interesting ninhydrin based one-pot four-component reaction which provides a simple and direct entry into a number of some new spiro [indeno[1,2-b]quinoxaline-11,3'-py rrolizidine] derivatives that may be of value in biological interest.

\section{Expelimental Section}

Synthesis of 2'-benzoyl-1',2',5',6',7',7'a-hexahydro-1'-phenylspiro[indeno[1,2-b]quinoxaline-11,3'-pynolizine] (5a) as a typical procedure. Proline $3(0.115 \mathrm{~g}$. $1 \mathrm{mmol})$ and chalcone ta $(0.2 \mathrm{lg} .1 \mathrm{mmol})$ were added to a solution of ninhydrin 1 $(0.178 \mathrm{~g} .1 \mathrm{~nm}$ ol) and 1,2-phenylenediamine $2 \mathrm{a}(0.108 \mathrm{~g} . \mathrm{l}$ 
mmol) in ethanol ( $5 \mathrm{~mL}$ ) and the mixture was refluxed for $2 \mathrm{~h}$ Progress of the reaction was monitored by T.L.C. using $n$-hexane/ ethyl acetate (10/5) mixture as eluent. The reaction mixture was cooled to $0^{\circ} \mathrm{C}$, the resulting solid was filtered off and washed with $3 \mathrm{ml}$ cooled ethanol (70\%) to give pure $5 \mathrm{a}$ in $85 \%$ yield. Cream solid: mp 167-169 ${ }^{\circ} \mathrm{C}$ : IR (KBr): ${ }^{2} 1695 \mathrm{~cm}^{-1} .{ }^{\mathrm{J}} \mathrm{H}$ NMR $\left(500 \mathrm{MHz}, \mathrm{CDCl}_{3}\right): \delta$ 1.92-2.2 $(\mathrm{m},+\mathrm{H}$. pyrrolizine). 2.56 (m. $1 \mathrm{H}$, pyrrolizine). 2.72 (m. $1 \mathrm{H}$. pyrrolizine). 4.24 (t. $J=$ $\left.10 \mathrm{~Hz}, 1 \mathrm{H} . \mathrm{H}^{1}\right), 4.51\left(\mathrm{dt}, J=9.6,6.7 \mathrm{~Hz} \cdot 1 \mathrm{H}, \mathrm{H}^{\prime \prime a}\right), 5.47(\mathrm{~d} . J=$ $\left.10 \mathrm{~Hz}, \mathrm{H}^{2}\right), 6.60-8.42$ (m. 18H. arom). ${ }^{13} \mathrm{C} \mathrm{NMR}(125 \mathrm{MHz}$. $\left.\mathrm{CDCl}_{3}\right): \delta 28.4,31.9,48.3,52.9,66.5 .73 .1 .75 .8 .122 .5 .127 .3$. $127.6,127.9 .128 .4,128.6,129.1,129.2,129.3,130.0,130.2$. $131.3,132.5 .137 .3,138.0 .140 .9 .142 .7,143.1 .144 .0,153.1$, 164.9. 198.2. MS: (mz) 493 (M+). 285. 256.218. Anal. Calcd. for $\mathrm{C}_{3} \mathrm{H}_{2}: \mathrm{N}_{3} \mathrm{O}:$ C. $82.73:$ H. 5.5 I: N. 8.51. Found: C. $82.80: \mathrm{H}$, 5.42: $\mathrm{N}, 8.41$. NOE (\%): irradiation of $\mathrm{H}^{\mathrm{l}^{\prime}}$ caused no considerable enhancement of $\mathrm{H}^{2}$ and $\mathrm{H}^{\circ}$. More purified products can be achieved by column chromatography on silica gel

Selected physical and spectroscopic data for isolated products. 2'-Benzoyl-7,8-dimethyl-1',2',5',6,7',7'a-hexahydo-1'phenyls]im [indeno[1,2-b]quinoxaline-11,3'-py molizine] (5b). Light yellow solid: $\operatorname{mp} 202-203^{\circ} \mathrm{C}$ (dec.): $\mathbb{R}$ (KBr): $\overline{I^{\circ}} 1697 \mathrm{~cm}^{-1}$. ${ }^{\mathrm{l}} \mathrm{H} \mathrm{NMR}\left(500 \mathrm{MHz} . \mathrm{CDCl}_{3}\right)$; ò 1.82-2.17 (m, 4 H. pyrolizine). 2.48 (m. $1 \mathrm{H}$. pyrrolizine). 2.52 (s. $\left.3 \mathrm{H}, \mathrm{CH}_{3}\right) .2 .57\left(\mathrm{~s} .3 \mathrm{H}, \mathrm{CH}_{3}\right.$ ). 2.67 (m. $1 \mathrm{H}$, py rrolizine). 4.18 (t. $J=10.2 \mathrm{~Hz} .1 \mathrm{H}, \mathrm{H}^{\mathrm{j}}$ ). 4.50 (dt. $J=9.4 .6 .7 \mathrm{~Hz}, 1 \mathrm{H} . \mathrm{H}^{\prime 2}$ ) $5.40\left(\mathrm{~d} . J=11.4 \mathrm{~Hz} . \mathrm{H}^{2}\right) \cdot 6.56-8.15$ (m. 16H arom). ${ }^{13} \mathrm{C} \mathrm{NMR}\left(125 \mathrm{MHz}, \mathrm{CDCl}_{3}\right): \hat{o} 20.3,20.4$. 27.8. 31.4. 48.0. 52.5. 65.9. 72.6. 75.2. 121.8, 126.9, 127.2 . $127.5,127.8,128.2,128.3,128.7,129.1,129.5,130.4,132.1$, $136.8,138.0 .139 .2,139.9 .140 .5 .141 .1,141.5 .143 .3,151.7$, 197.8. MS: (m/z) 521 (M) . 256.83. Anal Calcd for $\mathrm{C}_{36} \mathrm{H}_{31} \mathrm{~N}_{3} \mathrm{O}$ : C. $82.89 ;$ H, 5.99: N, 8.06. Found: C. 82.96: H, 6.09; N. 8. 18 . NOE $(\%)$ : irradiation of $\mathrm{H}^{\mathrm{l}^{\prime}}$ caused enhancement of $\mathrm{H}^{z^{\prime \prime}}(0.5 \%)$ and $\mathrm{H}^{\prime \prime a}(0.6 \%)$.

2'-Benzoyl-7,8-dimethyl-1',2',5',6;7,7'a-hexahydro-1'-(4methylphenyl)spiro[indeno[1,2-b]quinoxaline-11,3'-pynolizine] (5c). Cream solid; mp 193-195 ${ }^{\circ} \mathrm{C}: \mathbb{R}(\mathrm{KBr}): \overline{\mathrm{i}}^{7} 1695 \mathrm{~cm}^{-1} .{ }^{\mathrm{l}} \mathrm{H}$ NMR (500 MHz, $\mathrm{CDCl}_{3}$ ): ồ 1.89-2.19 (m, 4H. pyrrolizine). 2.36 (s. $\left.3 \mathrm{H}, \mathrm{CH}_{3}\right) .2 .52\left(\mathrm{~m} . \mathrm{lH}\right.$, pyrrolizine) 2.55 (s. $\left.3 \mathrm{H}, \mathrm{CH}_{3}\right)$. 2.61 (5. $3 \mathrm{H}, \mathrm{CH}_{3}$ ). 2.68 (m. $1 \mathrm{H}$, py rrolizine) 4.19 (t. $J=6.6$ $\left.\mathrm{Hz}, \mathrm{IH}, \mathrm{H}^{\mathrm{l}}\right) \cdot 4.53\left(\mathrm{ddd} J=16.5 \cdot 6.6 .6 .6, \mathrm{IH}, \mathrm{H}^{\prime 2}\right) .5 .42(\mathrm{~d} J=$ $\left.11.5 \mathrm{~Hz}, \mathrm{H}^{-}\right), 6.61-8.17$ (m. 15H. arom). ${ }^{13} \mathrm{C} \mathrm{NMR}(125 \mathrm{MHz}$. $\left.\mathrm{CDCl}_{3}\right): \hat{o} 20.7,20.8,21.5,28.3 .31 .8,48.3,52.6,66.4,73.0$. 75.7. 122.1. 127.6. 127.9, 128.3, 128.5, 128.6, 129.5, 129.7 . $129.8,130.8 .132 .4,136.8 .137 .3 .137 .9,138.3 .139 .5,140.2$, 141.5, 143.8, 152.2, 163.9, 198.3. MS: $(\mathrm{m} / \mathrm{z}) 535\left(\mathrm{M}^{+}\right), 284$. 246. Anal. Calcd. for $\mathrm{C}_{3}: \mathrm{H}_{33} \mathrm{~N}_{3} \mathrm{O}: \mathrm{C}, 82.96: \mathrm{H}, 6.21: \mathrm{N}, 7.84$. Found: C. 83.06: H. 6.10: N. 7.99. NOE (\%): irradiation of $\mathrm{H}^{\mathrm{j}^{\prime}}$ caused no considerable enhancement of $\mathrm{H}^{2 \prime}$ and $\mathrm{H}^{\prime \prime a}$.

2'-Benzoyl-1'-(4-fluorophenyl)-1',2',5',6,7',7'a-hexahydrospiro[indeno $[1,2-b] q u i n o x a l i n e-11,3$-pynolizine] (5d). Cream solid; mp $218-219^{\circ} \mathrm{C}$; IR (KBr): $\bar{I} 1692 \mathrm{~cm}^{-1}$. ${ }^{\mathrm{l}} \mathrm{H}$ NMR $(500$ $\mathrm{MHz}, \mathrm{CDCl}_{3}$ ): ò $1.89-2.19$ (m. $4 \mathrm{H}$. pyrrolizine). 2.36 (s. $3 \mathrm{H}$. $\mathrm{CH}_{3}$ ). 2.52 (m. $1 \mathrm{H}$, pyrrolizine), 2.55 (s, 3H, CH $) .2 .61$ (s. $\left.3 \mathrm{H}, \mathrm{CH}_{3}\right), 2.68(\mathrm{~m}, \mathrm{lH}$, pyrrolizine). $4.19(\mathrm{t}, J=6.6 \mathrm{~Hz}, \mathrm{lH}$. $\left.\mathrm{H}^{1}\right), 4.53$ (ddd. $\left.J=16.5 .6 .6,6.6 \mathrm{~Hz}, 1 \mathrm{H} . \mathrm{H}^{1}\right), 5.42(\mathrm{~d} . J=$ $\left.11.5 \mathrm{~Hz}, \mathrm{H}^{3}\right), 6.61-8.17$ (m. 15H. arom). ${ }^{13} \mathrm{C} \mathrm{NMR}(125 \mathrm{MHz}$. $\left.\mathrm{CDCl}_{3}\right): \hat{o} 13.8 .20 .6 .20 .7,29.5,33.0,34.1,47.2,57.2,60.2$.
$65.3,75.1 .122 .4,127.7,128.9,129.2 .130 .4 .131 .6 .138 .8$, $140.2,140.4,1+1.2,141.7,145.3,154.1,165.0,170.4$. MS $(\mathrm{m} / 2) 511\left(\mathrm{M}^{-}\right)$. 285. 218: Anal Calcd for $\mathrm{C}_{2} \mathrm{H}_{2} \mathrm{FN}_{3} \mathrm{O}: \mathrm{C}$. 79.82: H. 5.12: N. 8.21. Found: C, 79.69; H, 5.25: N. 8.29. NOE $(\%)$ : irradiation of $\mathrm{H}^{1}$ caused enhancement of $\mathrm{H}^{2}(1.5 \%)$ and $\mathrm{H}^{\mathrm{i} a}(1.2 \%)$

7,8-Dimethyl-2'-(4-fluombenzoyl)-1',2',5',6',7',7'a-hexahydro-1'-phenylspino[indeno[1,2-b]quinoxaline-11,3'-pynolizine] (5e). Light yellow solid: mp $164-165^{\circ} \mathrm{C}$ (dec.): IR (KBr): $\bar{r}$ $1698 \mathrm{~cm}^{-1}$. ${ }^{1} \mathrm{H} \mathrm{NMR}\left(500 \mathrm{MHz} . \mathrm{CDCl}_{3}\right): \delta$ o $1.90 .2 .01,2.16(\mathrm{~m}$ $4 \mathrm{H}$, pyrrolizine), $2.53\left(\mathrm{~s}, 3 \mathrm{H} . \mathrm{CH}_{3}\right), 2.54$ (n, $\mathrm{lH}$, pyrrolizine), $2.57\left(\mathrm{~s}, 3 \mathrm{H}, \mathrm{CH}_{2}\right), 2.65(\mathrm{~m}, \mathrm{lH}$, py rrolizine $) .49\left(\mathrm{~m}, 1 \mathrm{H}, \mathrm{H}^{1}\right)$. 4.51 (n. $\left.1 \mathrm{H}, \mathrm{H}^{\prime \prime}\right), 5.36$ (d. $\left.J=9 \mathrm{~Hz} \cdot \mathrm{H}^{\prime \prime}\right) .6 .27-8.13$ (m. $15 \mathrm{H}$, arom). ${ }^{12} \mathrm{C} N \mathrm{NM}\left(125 \mathrm{MHz}, \mathrm{CDCl}_{3}\right): \delta$ 19.8.19.9. 27.4. 30.8, 47.4. 52.0.65.4, 72.1,74.7, 121.3. 126.5, 127.4, 127.7, 128.2, $128.5,129.0,129.6 .129 .7,130.0 .132 .7,134.3,136.7 .136 .8$, $137.4,137.8,138.9 .139 .4,140.5,141.0,145.5,154.4,163.5$, 195.6. MS: $(\mathrm{m} / \mathrm{z}) 511\left(\mathrm{M}^{-}\right), 285(100), 256(70) .83$ (90). Anal. Calcd for $\mathrm{C}_{36} \mathrm{H}_{3}\left(\mathrm{FN} \mathrm{FN}_{3} \mathrm{O}: \mathrm{C} .80 .12: \mathrm{H}, 5.60: \mathrm{N}, 7.79\right.$ : Found: $\mathrm{C}$. 80.22: H. 5.52. N, 7.70. NOE (\%): irradiation of $\mathrm{H}^{1}$ caused enlancement of $\mathrm{H}^{2}(1.2 \%)$ and $\mathrm{H}^{\mathrm{F}^{\prime \prime}}(1.1 \%)$.

7,8-Dimethyl-2'-(4-fluolobenzoyl)-1'-(4-fluovophenyl)-1', 2',5',6',7',7'a-hexahỵdrospiro[indeno [1,2-b] (quinoxaline-11,3'pymolizine] (5f). Light yellow solid: $\operatorname{mp~} 167-169^{\circ} \mathrm{C}$ (dec.): $\mathbb{R}$ $(\mathrm{KBr}): \overline{i^{\prime}} 1746 \mathrm{~cm}^{-1}$. ${ }^{1} \mathrm{H}$ NMR $\left(500 \mathrm{MHz}, \mathrm{CDCl}_{3}\right)$ : ô $1.48-2.15$ (m. $4 \mathrm{H}$. pyrrolizine). 2.53 (s. $3 \mathrm{H}, \mathrm{CH}_{3}$ ). 2.54 (m. $\mathrm{lH}$. py rrolizine). 2.58 (s. $\left.3 \mathrm{H}_{1} \mathrm{CH}_{2}\right) .2 .65$ (1m, IH. pyrrolizine). 4.17 (t. $J=9.7$ Hz. $1 \mathrm{H}, \mathrm{H}^{\mathrm{l}}$ ), 4.48 (n. $\left.1 \mathrm{H}, \mathrm{H}^{\circ \prime \prime}\right), 5.36$ (d. $J=10.2 \mathrm{~Hz} . \mathrm{H}^{2}$ ), $6.27-8.13$ (m, l4H, arom). ${ }^{13} \mathrm{C}$ NMR (125 MHz, CDCl $): \delta$ $17.9,19.8,27.3 .30 .7,47.3 .51 .3 .65 .5,72.0 .74 .6,113.8$, 114.1. 114.9. 115.2. 121.4. 127.3. 127.8. 128.4 129.1. 129.2. $129.5,129.6,130.0 .132 .6,135.5 .137 .4,139.0,139.7 .140 .5$. $141.0,159.7,163.0,195.6$. MS: $(m / z) 557\left(\mathrm{M}^{-}\right)$. Anal. Calcd. for $\mathrm{C}_{36} \mathrm{H}_{2} \mathrm{~F}_{2} \mathrm{~N}_{3} \mathrm{O}:$ : C. 77.54: H. 5.24: N. 7.54. Found: C. 77.55: H. $5.32 ;$ N. 7.66. NOE (\%): irradiation of $\mathrm{H}^{1^{\prime}}$ caused no considerable enhancement of $\mathrm{H}^{2 \prime}$ and $\mathrm{H}^{* 1}$.

Acknowledgments. We gratefully acknowledge the financial support from the Research Council of Perșian Gulf University

\section{Refeiences}

1. (a) Tries, S.; Laufer, S. Inflammophamacologv 2001, 9, 113 ; (b) Argyropoulos, N. G:; Sarli, V. C: Gdaniec, M. Eun J. Org. Chem. 2006, 71, 3738; (c) Borsini, E.; Broggini, G.: Contini, A.; Zecchi, G. Eur. J. Org. Chem. 2008, 73, 2808, and references cited there in

2. (a) Sardina, F. T.; Rapoport, H. Chem. Rev, 1996, 96, 1825; (b) 1,3-Dipolar Cucloaddition Chemistm; Padwa, A., Ed; Wiley: New York, 1984: Vols. l and 2; (c) Tsuge, O.: Kanemasa, S. In Adwances in Heterocuclic Chentisty; Katritzky, A. R., Ed: Academic Press: San Diego, 1989: Vol. 45, p 231

3. (a) Gothelf, K. V.: Iorgensen. K. A. Chem. Rev. 1998, 98, 863; (b) Najera, C.; Sansano, G. M. Curr. Org. Chem. 2003, 7, 1105.

4. (a) Zhu, I: Bienayme, H. M/niti-component Reactions; VCH: Weinhein, Gemany, 2005; (b) Domling. A. Chem. Rev. 2006, 106.17

5. (a) Azizian, T.; Mohammadizadeh, M. R.; Zomorodbakhsh, S.; Mohammad, A. A.; Karimi, A. R. Arkivoc 2007, IT; 25; (b) Azizian. I: Mohammadizadeh. M. R:; Mohammad, A. A.; 
Karimi, A. R. Heteroatom (hem 2005, 16, $25 \%$ (c) Azizian, J.: Mohammadizadeh, M. R.: Mohanmad, A. A.; Karmi, A. R. J. Org. (Them. 2005, 70, 350): (d) Azizian, J.: Karimi, A. R.: Kazemizadeh, Z.; Mohammad, A. A.: Mohammadizadeh, M. R. Sinthesis 2005, 1095; (e) Azizian, J.: Karimi, A. R.: Kazemizadeh, 2.: Mohanmad, A. A.: Mohanunadizadeh, M. R. J. Org. Chem. $2005,70,1471$.

6. (a) Azizian, T.: Mohammadizadeh, M R.: Karimi, N.: Kazemizadeh, Z: Mohammad, A. A.; Karimi, A. R. Heteroatom Chem. 2005, 16, 549: (b) Azizian, T; Mohammadizadeh, M. R.: Mohanmmad, A. A.: Karimi, A. R.: Temour, F. Heteroatom (hem. 2007, 18, 16; (c) Azizian, T.: Karimi, A. R.; Mohammad, A. A.: Mohammadizadel, M. R. Symthesis 2004, 2263.
7. (a) Friedman, M. J. Agric. Food Chem 2004, 52, 385; (b) Hallnan, I. L.; Bartsch, R. A.J. Org. Chem. 1991, 56.6243: (c) McCaldin. D. J. Chem Rev. 1960, 60, 39; (d) Shapiro, R.; Chatterjie, N. J. Ong. Chem. 1970. 35.447: (e) Posner, G. H. Chem Rev 1986.86.831. 8. (a) Broggini, G.; Zecchi, G. Shnthesis 1999, 905; (b) Najera, C.; Sansano, T. M. Angew. Chem. Int. Ed. 2005, 4t,6272.

9. (a) Allway, P: Grigg, R. Tetrahedron Lett. 1991, 32, 5817: (b) Coulter, T.; Grigg, R.; Malone, J. F.; Sridharan, V. Tetrohedron Letr. 1991, 32, 5417: (c) Grigg, R.: Idle, I.; McMeekin, M.; Surendrakumar, S.; Vinod, D. J. Chem. Soc., Perkin Trans. I 1988, 2693; (d) Grigg, R:; Idle, T.; McMeekin, M.; Surendrakumar, S.: Vinod, D. J. Chem. Soc., Perlin Trans. 1 1988, 2703. 